\title{
Tártago asociado a cultivos anuales: una opción para la agricultura familiar
}

\author{
Castor bean intercropped with annual crops: an option for family farming
}

\author{
Jimmy Walter Rasche Alvarez ${ }^{1 *}$, Diego Augusto Fatecha Fois ${ }^{1}$, Nelson Gabriel Gaona Riego ${ }^{1}$, \\ José Francisco Ibarra Olhagaray ${ }^{1}$ y Gustavo Adolfo Rolón Paredes ${ }^{1}$ \\ ${ }^{1}$ Facultad de Ciencias Agrarias, Universidad Nacional de Asunción. San Lorenzo, Paraguay. \\ * Autor para correspondencia (jwrasche@yahoo.com.ar) \\ Recibido: 07/08/2014; Aceptado: 12/05/2015. \\ http://dx.doi.org/10.18004/investig.agrar.2015.junio.27-35
}

\section{RESUMEN}

El tártago por sus características agroecológicas se adapta a la agricultura familiar. Para evaluar la factibilidad de la asociación de tártago con cultivos anuales se instaló un experimento con diseño en bloques completos al azar, con siete tratamientos y tres repeticiones, en el Distrito de Escobar, Departamento de Paraguarí, entre agosto de 2007 y julio de 2009. Los tratamientos fueron T1: monocultivo de maíz; T2: monocultivo de poroto; T3: monocultivo de algodón; T4: monocultivo de tártago; T5: tártago asociado a maíz; T6: tártago asociado a poroto; T7: tártago asociado a algodón. Fueron analizadas altura del cacho primario, altura de plantas de tártago en el primer año y rendimiento en granos de tártago, maíz, poroto y algodón en rama, en monocultivo y asociados y la eficiencia de las asociaciones mediante el Índice Equivalente de la Tierra (IET) en los dos años. La mayor altura de inserción del cacho primario y altura final de plantas se obtuvieron en la asociación del tártago con maíz $(149,0 \mathrm{~cm})$, y de tártago con algodón $(215,0 \mathrm{~cm})$, respectivamente, evaluadas en el primer año de experimentación. El rendimiento de tártago en el mismo año en monocultivo y en asociación presentó escasa variación, entre 963 y $1059 \mathrm{~kg} \mathrm{ha}^{-1}$. En el segundo año, se obtuvo menor producción en la asociación de tártago con maíz (898 kg ha ${ }^{-1}$ ) y con algodón (890 kg ha') que en monocultivo $\left(1056 \mathrm{~kg} \mathrm{ha}^{-1}\right)$. El IET obtenido en los dos años indica que asociando el cultivo de tártago con algodón, maíz o poroto se logra un uso más eficiente del suelo.

Palabras clave: Ricinus communis L., asociación de cultivos, índice equivalente de la tierra, maíz, poroto, algodón.

\begin{abstract}
Castor bean Ricinus communis L., due to its agroecological features, is suitable for family farming. A randomized complete block test was carried out at the district of Escobar, department of Paraguari, Paraguay, between August 2007 and July 2009, in order to assess the feasibility of intercropping castor bean with annual crops, with seven treatments and three repetitions. The treatments were T1: maize monoculture; T2: bean monoculture; T3: cotton monoculture; T4: castor bean monoculture; T5: Castor bean intercropped with maize; T6: Castor bean intercropped with bean; T7: castor bean intercropped with cotton. The following were measured: Primary bunch height, castor bean plant height in the first year and castor bean, maize, beans and cotton yield, both in monoculture and in association with other crops, as well as the association efficiency measured with the Land Equivalent Ratio (LER) in the two years. The highest peaks of primary branch height and the final height of plants were obtained in castor bean in association with maize $(149 \mathrm{~cm})$ and castor bean in association with cotton $(215 \mathrm{~cm})$ respectively, assessed in the first year of the study. Castor bean yield in monoculture and intercropped during the same year showed slight variation, ranging between 963 and $1059 \mathrm{~kg}$ ha -1 . In the second year, lower production was obtained in castor bean intercropped with maize (898 kg ha-1) and with cotton (809 kg ha-1) than that obtained with monoculture (1056 kg ha-1). LER results in the two years showed that castor bean intercropped with cotton, maize or bean, leads to a more efficient land use.
\end{abstract}

Key words: Ricinus communis L., intercropped systems, land equivalent ratio, maize, beans, cotton. 


\section{INTRODUCCIÓN}

En el Paraguay, el tártago (Ricinus communis L.) ocupa un área de aproximadamente 5.500 ha (DCEA/MAG 2013), es sembrado principalmente dentro de la agricultura familiar, el $78 \%$ de la superficie de tártago es sembrado por pequeños productores, cuyas propiedades varían entre 1 a 20 ha de superficie, situados especialmente en los departamentos de San Pedro y Concepción (DCEA/MAG 2009), representando inclusive en algunos municipios, una de las principales fuentes de renta, como es el caso del distrito de Choré (Flores y Maldonado 2007). Sin embargo, durante el periodo que abarca de 2006 a 2013, se registró una disminución de hasta el $50 \%$ del área sembrada de este rubro (DCEA/MAG 2006, DCEA/MAG 2013).

Las causas de la reducción del área de siembra pueden ser atribuidas, entre otros factores, al bajo precio de venta de los granos, los altos costos de producción por el requerimiento de mano de obra, principalmente para el control de malezas y la cosecha del cultivo, y el lento crecimiento del cultivo en su fase inicial (Paulo et al. 1997; Azevedo et al. 2006, Costa et al. 2014). Otras causas pueden ser atribuidas al deterioro del suelo luego de algunos años de uso, acompañado de la alta tasa de erosión hídrica, por permanecer sin cobertura en gran parte del año, siendo que el tártago es el cultivo que más favorece a la pérdida de suelo y agua, debido a la baja protección que ofrece contra los factores erosivos (Bertoni et al. 1976).

Una de las alternativas que el productor de tártago puede considerar ante tales situaciones, es la de asociar el cultivo con otros de ciclo más corto y con mayor densidad de siembra, pudiendo de esta manera disminuir el costo de producción, debido a la reducción del control de malezas, y además, contar con la posibilidad de obtener otros ingresos económicos entre la siembra y cosecha del tártago (Furtado et al. 2014).

La asociación de cultivos es una práctica común utilizada por productores que poseen escasa área disponible para producir, principalmente en zonas tropicales y subtropicales (Bezerra Neto y Robichaux 1996, Bezerra Neto y Robichaux 1997, Pinto et al. 2011), donde los procesos de descomposición de la materia orgánica son más acelerados, siendo una buena alternativa para mantener una mayor estabilidad de cobertura muerta en el suelo (Calegari 2007).
Uno de los principios para la asociación de cultivos es la complementariedad de los mismos, siendo mejor cuanto más diferentes sean las especies involucradas, por lo que se recomienda asociar cultivos de diferentes familias (Teixeira et al. 2012, Furtado et al. 2014). Otro aspecto interesante de la asociación es que al permitir mayor densidad de plantas por unidad de área, se obtiene mayor cobertura del suelo, reduciendo la incidencia de malezas, aumentando la estabilidad del sistema de producción frente a la diversidad de estaciones climáticas y una compensación de la producción gracias a la diversificación (Zaffaroni 1987), por lo que este sistema permitiría eliminar los problemas de cobertura y erosión del suelo ocasionado por el monocultivo del tártago.

De esta manera, las asociaciones de cultivos pueden constituirse en una tecnología de fácil aplicación para productores familiares, ya que el objetivo del mismo es maximizar los recursos disponibles como el área aprovechable, mano de obra, insumos, además de minimizar los riesgos de pérdidas de la producción, mediante la diversificación de rubros. Sin embargo, uno de los grandes desafíos es determinar cuáles pueden ser las asociaciones más convenientes (Rezende et al. 2005).

Entre los índices utilizados para comparar los sistemas de cultivos asociados y monocultivos, está el índice equivalente de la tierra (IET) definido por Willey (1979) como el área relativa de suelo bajo monocultivo que es requerido para proporcionar los rendimientos alcanzados en una asociación de cultivos. Cuando el valor del IET es igual a la unidad existe indiferencia en la asociación de los cultivos involucrados, mientras que si es mayor a la unidad indica efecto de sinergismo positivo en la asociación, habiendo ventajas en la misma y cuando es menor a la unidad ocurre antagonismo o competencia entre la asociación, por lo que la misma presenta desventajas frente al monocultivo (Teixeira et al. 2005).

Con relación al tártago, existen varios trabajos que muestran que el mismo puede asociarse a ciertos cultivos como maíz (Zea mays L.) (Savy Filho et al. 1989, Azevedo et al. 1998a, Azevedo et al. 2007, Azevedo et al. 2001, Pinto et al. 2011, Francelino et al. 2013), poroto (Phaseolus vulgaris L.) (Teixeira et al. 2011, Teixeira et al. 2012), habilla (Vigna unguiculata L.) (Távora et al. 1988, Savy Filho et al. 1989, Rando y Quintanilha 1990, Corrêa et al. 2006, Pinto et al. 2011, Souza Jr. et al. 2012, Francelino et al. 2013, Furtado et al. 2014), sorgo (Sorghum bicolor (L.) Moench) (Azevedo et al. 1998a, Azevedo et al. 1998b, Corrêa et al. 2006), maní (Arachis 
hypogaea L.) (Távora et al. 1988), sésamo (Sesamum indicum L.) (Beltrão et al. 2010, Souza Jr et al. 2012, Magalhães et al. 2013) y abonos verdes como el cuandú (Canavalia ensiformis (L.) DC.), mucuna enana (Stizolobium deeringianum Steph y Bart.) (Rando y Quintanilha 1990).

El objetivo de esta investigación fue verificar la factibilidad de la asociación de tártago con maíz, poroto y algodón por medio del índice equivalente de la tierra (IET) en dos ciclos de producción e identificar la mejor asociación entre los cultivos.

\section{MATERIALES Y MÉTODOS}

El experimento se llevó a cabo en una finca agropecuaria situada en la localidad de Ybyraty, Distrito de Escobar, en el Dpto. de Paraguarí, en el periodo comprendido entre agosto de 2007 y julio de 2009. El terreno utilizado posee una pendiente entre 3 a $8 \%$ y el suelo es de textura arenofranca en la superficie y clasificado como Alfisol según López et al. (1995).

El análisis químico de la camada superficial del suelo (0 $0,20 \mathrm{~m})$, realizado antes de la instalación del experimento presentó los siguientes resultados: $\mathrm{pH}=5,3$; Mat. Org.= $0,76 \% ; \mathrm{P}=1,0 \mathrm{mg} \mathrm{dm}{ }^{-3} ; \mathrm{Ca}^{+2}=0,43 \mathrm{cmol}_{\mathrm{c}} \mathrm{kg}^{-1} ; \mathrm{Mg}^{+2}=$ $0,13 \mathrm{cmol}_{\mathrm{c}} \mathrm{kg}^{-1} ; \mathrm{K}^{+}=0,06 \mathrm{cmol}_{\mathrm{c}} \mathrm{kg}^{-1} ; \mathrm{Al}^{+3}+\mathrm{H}^{+}=0,26$ $\mathrm{cmol}_{\mathrm{c}} \mathrm{kg}^{-1}$. Según Loncharich (2008), el clima del lugar es subtropical moderado, la temperatura media anual es de $22,3^{\circ} \mathrm{C}$ y la precipitación media anual es de $1450 \mathrm{~mm}$. La precipitación mensual y días de precipitación durante cada mes que abarcó el experimento, se puede observar en la figura 1.

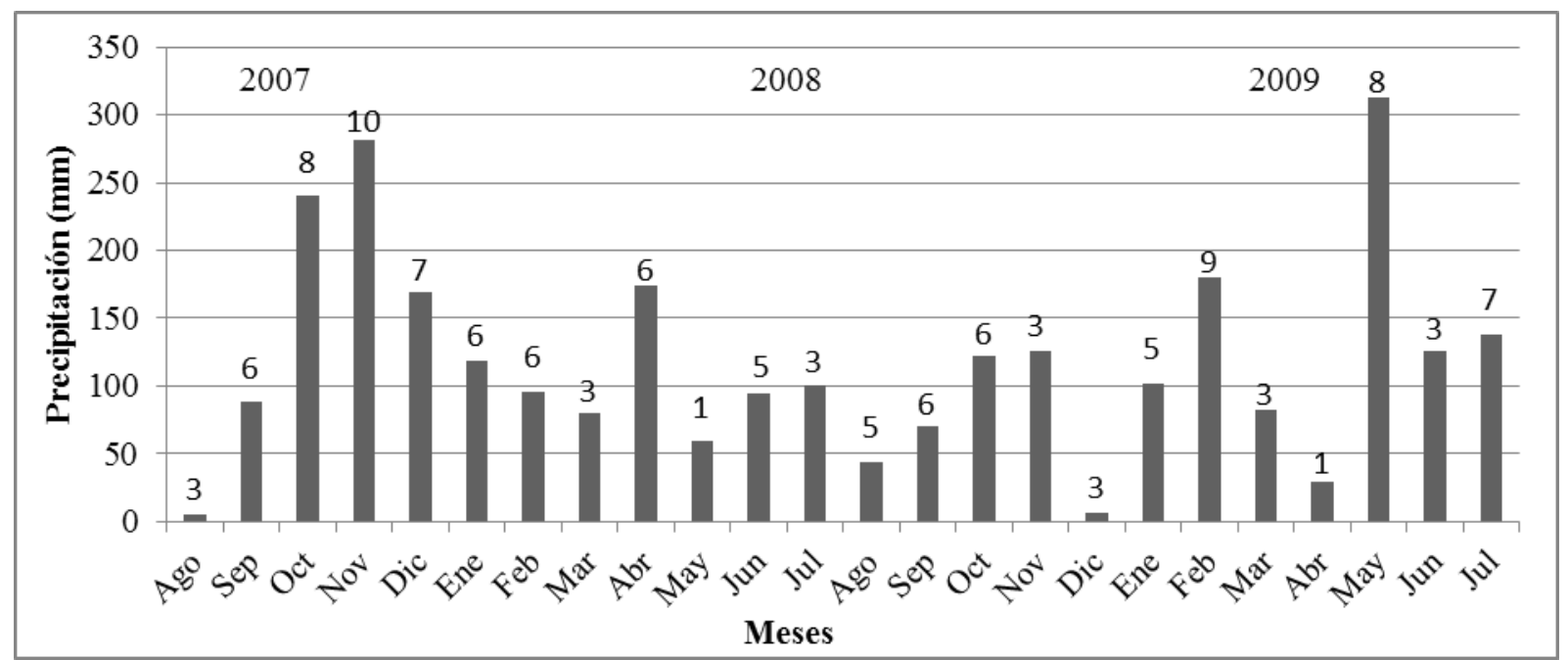

Figura 1. Histórico de precipitación mensual ( $\mathrm{mm}$ ) y días de precipitación en cada mes, ocurridos desde agosto de 2007 hasta julio de 2009 en la estación meteorológica de Paraguarí, Paraguarí. Fuente: Mundomanz 2014.

El diseño experimental utilizado fue de bloques completos al azar, con siete tratamientos $y$ tres repeticiones. Los tratamientos fueron $\mathrm{T} 1$ : monocultivo de maíz; T2: monocultivo de poroto; T3: monocultivo de algodón; T4: monocultivo de tártago; T5: tártago asociado a maíz; T6: tártago asociado a poroto y T7: tártago asociado a algodón.

La superficie del experimento fue de $1822 \mathrm{~m}^{2}(67,5 \mathrm{~m} \mathrm{x}$ $27 \mathrm{~m}$ ) la cual fue dividida en 21 parcelas de $67,5 \mathrm{~m}^{2}(7,5$ m x $9 \mathrm{~m}$ ). Cada unidad experimental estuvo conformada por tres hileras de tártago con 5 plantas por hilera, obteniéndose 15 plantas por unidad experimental.
En el primer año del experimento, en julio de 2007, se aplicaron $2 \mathrm{t} \mathrm{ha}^{-1}$ de cal agrícola, y se procedió a la incorporación de la misma mediante una rastreada profunda. Fueron aplicados $2 \mathrm{~L} \mathrm{ha}^{-1}$ de herbicida Glyphosato 48\%, dos semanas antes de la implantación del experimento, que fue realizada en la última semana de octubre de 2007.

Antes de realizar la siembra de los cultivos, se realizó una fertilización de base aplicando 20; 50 y $40 \mathrm{~kg} \mathrm{ha}^{-1}$ de $\mathrm{N}$; $\mathrm{P}_{2} \mathrm{O}_{5}$ y $\mathrm{K}_{2} \mathrm{O}$, respectivamente, mediante la aplicación de $110 \mathrm{~kg} \mathrm{ha}^{-1}$ de fosfato di amónico (18-46-00) y $65 \mathrm{~kg} \mathrm{ha}^{-1}$ de cloruro de potasio (00-00-60) en la línea de siembra de 
los cultivos y en el hoyo de las plantas de tártago. Posteriormente, a los 40 días después de la siembra, se aplicaron $30 \mathrm{~kg} \mathrm{ha}^{-1}$ de $\mathrm{N}$ al voleo, en forma de urea (67 $\left.\mathrm{kg} \mathrm{ha}{ }^{-1}\right)$. La fertilización se realizó en base a la recomendación de Fatecha (2001).

La variedad de tártago sembrada fue IAC 80, con frutos dehiscentes, de porte intermedio, cuyas semillas madres son originarias del Instituto Agronómico de Campinas (IAC) y multiplicadas en el Centro Experimental Hernando Bertoni del Instituto Paraguayo de Tecnología Agraria (CEHB-IPTA). En las parcelas donde fue sembrado el tártago, se utilizó distanciamiento de $1,5 \mathrm{~m}$ entre plantas y $3 \mathrm{~m}$ entre hileras con una planta por hoyo (2.222 plantas por hectárea).

La variedad de maíz utilizada fue Karapé pyta, con distanciamiento en monocultivo de $0,50 \mathrm{~m}$ entre plantas y 0,75 m entre hileras, con dos plantas por hoyo (53.333 plantas por hectárea). En el tratamiento asociado al tártago se sembraron ocho hileras: tres hileras de maíz entre cada hilera de tártago y una hilera en cada extremo de la parcela; las hileras de maíz estuvieron a 0,80 m de las hileras de tártago y a $0,70 \mathrm{~m}$ entre ellas; en cada hoyo se dejaron dos plantas, equivalente a 42.667 plantas por hectárea.

La variedad de algodón sembrada fue la Guazuncho; con el mismo distanciamiento que en el caso del maíz, tanto para monocultivo como para cultivo asociado, por lo tanto se obtuvo la misma cantidad de plantas por parcela y por hectárea.

La variedad de poroto utilizada fue la Pyta'i, el distanciamiento en monocultivo fue de $0,3 \mathrm{~m}$ entre plantas y $0,75 \mathrm{~m}$ entre hileras, con 2 plantas por hoyo, equivalente a 88.888 plantas por hectárea. Para el caso de las parcelas asociadas, las hileras de poroto estuvieron a $0,80 \mathrm{~m}$ de las hileras de tártago y a $0,30 \mathrm{~m}$ entre sí, con un total ocho hileras de poroto, dos a cada extremo de la parcela y seis entre medio de las hileras de tártago (tres a cada lado de la hilera central), es decir, 71.000 plantas por hectárea.

La siembra de todos los cultivos, tanto en asociación como en monocultivo fueron realizados en el mismo día que del tártago, en ambos años de evaluación. En el primer año los cultivos fueron sembrados en octubre de 2007 y en el segundo año en octubre de 2008. En junio de 2008, fueron eliminadas las plantas de tártago y de algodón mediante una rastreada superficial y fue sembrada avena negra (Avena strigosa L.) al voleo en toda el área del experimento.

Posteriormente en el segundo año de experimentación, fue desecada la avena negra con Glyphosato y se procedió a sembrar, en la última semana de octubre de 2008, nuevamente los mismos cultivos, en las mismas distancias, y en la misma área, aplicando la misma cantidad de fertilizantes químicos, de acuerdo a cada tratamiento establecido en el primer año, con excepción de la cal agrícola.

Los cuidados fitosanitarios y control de malezas se realizaron según las necesidades del cultivo y especificaciones técnicas. Realizándose seis y dos carpidas en el monocultivo y cultivo asociado, respectivamente en el primer año y cuatro y dos carpidas en el segundo año.

La cosecha del tártago se llevó a cabo cuando los racimos se encontraban secos en un 70 a $80 \%$, debido a que la variedad IAC 80 es dehiscente, utilizándose tijera de podar para cortar los racimos, que posteriormente fueron expuestos a la luz solar por aproximadamente una semana, para su secado y apertura natural, luego fueron embolsados los granos correspondientes a cada unidad experimental, realizándose el pesaje con una balanza de precisión de dos dígitos decimales.

Para los cultivos de maíz y poroto se determinaron solamente los rendimientos de granos, y para algodón el rendimiento de fibra bruta, mediante pesaje con una balanza de precisión de dos dígitos decimales, tanto para el primer y segundo año.

En el primer año además del rendimiento se determinaron la altura de inserción del cacho primario del tártago, con cinta métrica, midiendo desde la base de la planta hasta la inserción en el tallo del cacho primario y la altura final desde el suelo hasta la parte más alta de la planta, siendo evaluadas cinco plantas de tártago por parcela.

Para el análisis del rendimiento, fueron evaluadas todas las plantas de cada unidad experimental tanto del primer como del segundo año. Los datos de las variables, fueron analizados estadísticamente mediante un análisis de varianza (ANAVA), y cuando los efectos de los tratamientos fueron significativos $(p<0,05)$ se realizó comparaciones de medias, utilizando el test de Tukey al $5 \%$. 
La evaluación de la eficiencia de los monocultivos y de los cultivos asociados fue realizada a través de la determinación del Índice Equivalente de la Tierra (IET), parcial y total. El primero correspondió al valor de cada cultivo en la misma parcela, mientras que para el IET total se realizó la suma de los valores de IET parciales. El IET fue calculado utilizando la siguiente fórmula:

$$
\mathrm{IET}=\mathrm{Px} / \mathrm{Mx}+\mathrm{Py} / \mathrm{Uy},
$$

donde: Px es el rendimiento del cultivo $x$ en policultivo; Mx es el rendimiento del cultivo $x$ en monocultivo; Py es el rendimiento del cultivo $y$ en policultivo; Uy es el rendimiento del cultivo $y$ en monocultivo (Teixeira et al. 2005).

\section{RESULTADOS Y DISCUSIÓN}

En el primer año de evaluación la altura de la inserción del cacho primario del tártago fue mayor $(149,0 \mathrm{~cm})$ cuando asociado al cultivo de maíz, comparado al tártago en monocultivo $(138,3 \mathrm{~cm})$, mientras que las demás asociaciones no presentaron diferencias significativas con el monocultivo (Tabla 1). Probablemente a consecuencia del crecimiento acelerado del maíz (planta C4), el tártago empleó más energía para el crecimiento de la planta en altura, a fin de un mejor aprovechamiento de la energía solar, quedando así con una mayor altura la inserción del primer cacho, ya que el mismo generalmente es una de las variables que más influye en el aumento de rendimiento.

Tabla 1. Altura de inserción del cacho primario y altura final de la planta de tártago en monocultivo y asociado a cultivos anuales en el primer año.

\begin{tabular}{lcc}
\hline & \multicolumn{2}{c}{ Altura (cm) } \\
\hline Cacho primario & Final de la planta \\
\hline Monocultivo de Tártago & $138,3 \quad \mathrm{~b}^{*}$ & $184,6 \mathrm{~b}$ \\
Tártago asociado a Maíz & $149,0 \mathrm{a}$ & $205,3 \mathrm{ab}$ \\
Tártago asociado a Poroto & $140,7 \mathrm{ab}$ & $196,8 \mathrm{ab}$ \\
Tártago asociado a Algodón & $145,7 \mathrm{ab}$ & $215,8 \mathrm{a}$ \\
\hline CV $(\%)$ & 2,2 & 4,3 \\
\hline
\end{tabular}

* Letras diferentes en las columnas significan diferencia estadística entre los tratamientos según prueba de Tukey al $5 \%$.

La importancia de la inserción del cacho primario radica en que la misma es responsable de gran parte de la producción de granos cuando el tártago es asociado a otros cultivos. En ese sentido, Francelino et al. (2013) observaron que la producción de granos en el racimo primario del tártago, cuando asociado al maíz, representa entre el 65 a $85 \%$ de la producción total, sin embargo, en monocultivo representa solamente el $38 \%$ de la producción total de granos de tártago. Igualmente, Furtado et al. (2014) observaron correlación positiva entre altura del cacho primario con el rendimiento de tártago en el cultivar IAC 2028 .

En relación a la altura final de la planta de tártago, este fue superior en la asociación con algodón $(215,8 \mathrm{~cm})$ que en el monocultivo $(184,6 \mathrm{~cm})$, pero no fue diferente de las demás asociaciones (Tabla 1).

Las plantas de algodón también desarrollaron un porte erecto de crecimiento, lo que probablemente provocó una competencia inicial con el tártago, forzando a que este crezca en altura, a fin de poder acceder a una mayor luminosidad, contraponiéndose al efecto esperado, donde la asociación con este cultivo anual afectó positivamente la altura del tártago. Esto no coincide con lo mencionado por Costa et al. (2014) quienes constataron que a mayor competencia de malezas dentro de los primeros 120 días de la siembra del tártago, es menor la altura del cultivo.

Así mismo, Araujo et al. (2004) y Magalhães et al. (2013) encontraron que la altura del tártago fue afectada por la presencia del cultivo de sésamo, disminuyendo la competencia a medida que había mayor diferencia de días entre la siembra del tártago y la del sésamo, ya que el crecimiento del tártago inicialmente es muy lento.

El rendimiento del tártago no presentó diferencia estadística significativa comparando el primer y segundo año de estudio, sea en monocultivo o asociado (Tabla 2).

Tabla 2. Rendimiento del tártago en monocultivo y asociado a los cultivos de maíz, poroto y algodón en las zafras agrícolas 2007/08 y 2008/09.

\begin{tabular}{lcc}
\hline \multicolumn{1}{c}{ Tratamientos } & \multicolumn{1}{c}{ Rendimiento del tártago $\left(\mathbf{k g ~ h a}^{\mathbf{- 1}}\right)$} \\
\hline Monocultivo de Tártago & Año I & Año II \\
Tártago asociado a Maíz & $1.059 \mathrm{Aa}^{*}$ & $1.056 \mathrm{Aa}$ \\
Tártago asociado a Poroto & $963 \mathrm{Aa}$ & $898 \mathrm{Ab}$ \\
Tártago asociado a Algodón & $1.160 \mathrm{Aa}$ & $1.013 \mathrm{Aa}$ \\
\hline CV (\%) & $1.012 \mathrm{Aa}$ & $890 \mathrm{Ab}$ \\
\hline
\end{tabular}

* Letras mayúsculas corresponden a las líneas, comparando la producción de tártago entre los años; letras minúsculas corresponden a las columnas, comparando la producción de tártago en monocultivo y en forma asociada en cada año según prueba de Tukey al $5 \%$. 
En el primer año, el rendimiento de tártago en monocultivo (1.059 $\mathrm{kg} \mathrm{ha}^{-1}$ ) y asociado a los cultivos de maíz (963 kg ha $\left.{ }^{-1}\right)$, de poroto $\left(1.160 \mathrm{~kg} \mathrm{ha}^{-1}\right)$ y de algodón (1.012 $\mathrm{kg} \mathrm{ha}^{-1}$ ), fueron similares (Tabla 2), sin embargo en el segundo año la producción de tártago en monocultivo (1.056 kg ha' $\mathrm{kge}^{-1}$ superior a la asociación con maíz (898 kg ha $\left.{ }^{-1}\right)$ y con algodón $\left(890 \mathrm{~kg} \mathrm{ha}^{-1}\right)$, no así cuando se asoció con poroto $\left(1.013 \mathrm{~kg} \mathrm{ha}^{-1}\right)$.

Se puede observar una disminución en el rendimiento del tártago al ser asociado, como consecuencia del aumento de competencia por luz, agua y nutrientes. En el caso del maíz hay que considerar que la misma es una planta de metabolismo fotosintético $\mathrm{C} 4$, con crecimiento inicial rápido, mayor índice foliar, arquitectura de planta y altura más propicia a la captación de luz solar, siendo más competitiva que el tártago, que es de metabolismo fotosintético $\mathrm{C} 3$, por lo que es esperado que cause disminución en el rendimiento del mismo, como ocurrió en el segundo año de evaluación. En ese sentido, es importante que en las asociaciones de tártago con cultivos, se debe priorizar la combinación con especies de bajo porte para no competir con el tártago, principalmente por luz solar (Sousa Jr. et al. 2012).

En el segundo año, en el mes de noviembre, y principalmente diciembre deł 2008 se registró escasa precipitación (Figura 1), lo que pudo afectar el rendimiento del tártago asociado, al haber una mayor competencia por agua en ese momento.
Existen varios trabajos demostrando el efecto del rendimiento del tártago cuando este es asociado a otros cultivos, entre los que se puede mencionar, Azevedo et al (2001) quienes observaron que a medida que se aumentaba la densidad de maíz en la asociación con tártago, había disminución del rendimiento del mismo. Igualmente, Corrêa et al. (2006) encontraron menor rendimiento del tártago cuando fue asociado con sorgo (489 $\mathrm{kg} \mathrm{ha}^{-1}$ ) que en monocultivo (960 kg ha ${ }^{-1}$ ), sin embargo, al igual que en el presente experimento, la asociación del cultivo con poroto no causó disminución en el rendimiento (774 $\mathrm{kg} \mathrm{ha}^{-1}$ ). Por otro lado, Morgado (2004), observó que el tártago asociado al poroto presentó mejora en el rendimiento, pasando de $2.101 \mathrm{~kg} \mathrm{ha}^{-1}$ en monocultivo, a $2.714 \mathrm{~kg} \mathrm{ha}^{-1}$.

En el segundo año de estudio el suelo ya se encontraba corregido con cal agrícola y fertilizado, sembrándose avena negra durante el invierno a fin de mejorar la cobertura del suelo, por lo cual la siembra de los cultivos fue realizada en labranza mínima, sin remoción del suelo, sin embargo el rendimiento del tártago fue similar al del primer año.

El Índice Equivalente de la Tierra (IET) obtenido en las diferentes asociaciones de tártago con maíz, poroto y algodón en el primer (Tabla 3) y segundo año (Tabla 4) indican su factibilidad de asociación.

Tabla 3. Rendimiento de los cultivos, e Índice Equivalente de la Tierra (IET) parcial y total de los monocultivos y cultivos asociados correspondiente al Año I

\begin{tabular}{|c|c|c|c|}
\hline \multirow[b]{2}{*}{ Tratamiento } & \multicolumn{3}{|c|}{ Año I } \\
\hline & $\begin{array}{c}\text { Rendimiento } \\
\left(\mathrm{kg} \mathrm{ha}^{-1}\right)\end{array}$ & $\begin{array}{c}\text { IET } \\
\text { parcial }\end{array}$ & $\begin{array}{l}\text { IET } \\
\text { total }\end{array}$ \\
\hline Monocultivo de maíz & 2.699 & 1,00 & $1,00 \quad b^{*}$ \\
\hline Monocultivo de poroto & 1.629 & 1,00 & $1,00 \mathrm{~b}$ \\
\hline Monocultivo de algodón & 1.061 & 1,00 & $1,00 \mathrm{~b}$ \\
\hline Monocultivo de tártago & 1.059 & 1,00 & $1,00 \mathrm{~b}$ \\
\hline Tártago asociado a maíz & $963+2.255$ & $0,91+0,89$ & $1,80 \mathrm{a}$ \\
\hline Tártago asociado a poroto & $1.160+741$ & $1,08+0,46$ & $1,54 \mathrm{ab}$ \\
\hline Tártago asociado a algodón & $1.012+321$ & $0,98+0,33$ & $1,31 \mathrm{ab}$ \\
\hline $\mathrm{CV}(\%)$ & & & 17,80 \\
\hline
\end{tabular}

*Letras diferentes en las columnas representan diferencia estadística entre los tratamientos según prueba de Tukey al 5\%.

En el primer año de experimentación se logró una utilización más eficiente del suelo asociando tártago con maíz, poroto y algodón, para lo cual son necesarios $80 \%$, $54 \%$ y $31 \%$ más de superficie de los respectivos monocultivos para alcanzar lo obtenido cuando son asociados. En el caso del tártago, además de no disminuir su rendimiento (Tabla 3), permite que se produzca casi la misma cantidad de maíz que se obtendría u obtiene en 
monocultivo, sin embargo el poroto y algodón tuvieron rendimientos inferiores de 46 y 33\%, comparados con su producción en monocultivos (Tabla 3). Aunque no existe diferencia estadística entre el IET del monocultivo del tártago y su asociación con poroto y algodón en el primer año, puede considerarse como una práctica agrícola recomendada para el pequeño productor, principalmente por su bajo costo económico (Zaffaroni 1987).

Con la asociación de cultivos, aparte de conseguir beneficios en la producción y diversificación de rubros agrícolas, por parte de pequeños productores, se pueden obtener beneficios indirectos, como una mejor utilización del recurso suelo, disminuyendo pérdidas por erosión hídrica, un mayor porcentaje de cobertura y reciclaje de nutrientes por aumento en la producción de material orgánico, y un mejor control de malezas, lo que permite obtener un menor costo de producción del cultivo principal, en este caso el tártago.

En el segundo año de evaluación, el IET por cultivo y el IET total fueron similares al primer año (Tabla 4), con una leve disminución en los rendimientos de los cultivos, debido a que durante ese año hubieron periodos de escasez hídrica (Figura 1), sin embargo no fue perjudicial, probablemente como consecuencia de las prácticas edáficas realizadas inicialmente, que aumentaron la cobertura del suelo y el crecimiento radicular, permitiendo que las plantas puedan desarrollarse y obtener rendimientos casi similares al del primer año.

Tabla 4. Índice Equivalente de la Tierra (IET) de los monocultivos, de cada cultivo cuando asociado (IET parcial), y el IET total, considerando ambos cultivos. Año II.

\begin{tabular}{|c|c|c|c|}
\hline \multirow[b]{2}{*}{ Tratamiento } & \multicolumn{3}{|c|}{ Año II } \\
\hline & $\begin{array}{c}\text { Rendimiento } \\
\left(\mathrm{kg} \mathrm{ha}^{-1}\right)\end{array}$ & IET por cultivo & IET total \\
\hline Monocultivo de maíz & 2.058 & 1,00 & $1,00 \mathrm{~b}^{*}$ \\
\hline Monocultivo de poroto & 1.346 & 1,00 & $1,00 \mathrm{~b}$ \\
\hline Monocultivo de algodón & 857 & 1,00 & $1,00 \mathrm{~b}$ \\
\hline Monocultivo de Tártago & 1.056 & 1,00 & $1,00 \mathrm{~b}$ \\
\hline Tártago asociado a maíz & $898+1629$ & $0,85+0,79$ & $1,64 \mathrm{a}$ \\
\hline Tártago asociado a poroto & $1.013+759$ & $0,96+0,56$ & $1,52 \mathrm{a}$ \\
\hline Tártago asociado a algodón & $890+464$ & $0,84+0,54$ & $1,38 \mathrm{ab}$ \\
\hline CV $(\%)$ & & & 8,3 \\
\hline
\end{tabular}

* Letras diferentes en la columna representan diferencia estadística entre los tratamientos según prueba de Tukey al 5\%.

El IET obtenido en la asociación de tártago con maíz, considerando la media del primer y segundo año fue de 1,72, superior a lo encontrado por Azevedo et al. (1998a) que obtuvieron un IET de 1,25 utilizando 5.000 plantas ha $^{-1}$ de tártago y 5.000 plantas ha $^{-1}$ de maíz, dando preferencia a una mayor producción de tártago, en cambio cuando se utilizaron 5.000 plantas ha ${ }^{-1}$ de tártago y 20.000 plantas ha ${ }^{-1}$ de maíz, teniendo mayor producción de maíz, obtuvieron un IET de 1,6. Azevedo et al. (2001) observaron que el tártago asociado con maíz puede alcanzar un IET próximo a 1,6 cuando el mismo es sembrado en alta densidad (5.000 plantas ha ${ }^{-1}$ ) y el maíz con densidad entre 5.000 a 20.000 plantas ha ${ }^{-1}$.

El IET obtenido en la asociación de tártago con poroto, considerando la media de los dos años fue de 1,53, superior al obtenido por Corrêa et al. (2006), quienes con la misma asociación de cultivos lograron un IET de 1,45. Por su parte Tavora et al. (1988), realizando la misma asociación obtuvieron un IET de 1,20. En cambio Azevedo et al. (1998a) lograron un IET de 1,85 utilizando 5.000 plantas ha ${ }^{-1}$ de tártago y 20.000 plantas $\mathrm{ha}^{-1}$ de poroto.

EL IET promedio obtenido al asociar tártago con algodón fue de 1,35 siendo inferior a las demás asociaciones, sin embargo tiene ventaja en la producción sobre el monocultivo, esto sin considerar los beneficios causados por la asociación de cultivos desde el punto de vista edáfico y de mano de obra.

\section{CONCLUSIONES}

El rendimiento de tártago en monocultivo y asociado es similar en el primer año de evaluación, sin embargo, en el segundo año cuando asociado al maíz y al algodón presenta menor rendimiento. 
Según el Índice Equivalente de la Tierra la mejor combinación, para aumentar los rendimientos es la asociación de tártago con maíz. Mientras que las asociaciones de tártago con maíz, poroto y algodón permiten la utilización más eficiente del suelo, que en monocultivo.

\section{REFERENCIAS BIBLIOGRÁFICAS}

Araújo F, JOT; Beltrão, NE de M; Moura, MF de; Viana, J; Bruno, GB; Bruno, R de LA. 2004. Época de plantio do gergelim em consorcio com a mamona. In: Congreso Brasilero de Mamona (1, 2004, Campina Grande, BR). Trabajos presentados. Campina Grande, BR: UFPB/CCA, EMBRAPA/CNPA. 4 p.

Azevedo, DMP de; Beltrão, JWS; Vieira, D J; Nóbrega, LB da; Lima, EF; Batista, FAS; Pereira, JR. 1998a. Efeito de população de plantas no rendimento do consórcio de mamona com culturas alimentares. Revista de Oleaginosas e Fibrosas 2(3):193-202.

Azevedo, DMP de; Beltrão, JWS; Santos, JW dos; Lima, EF; Batista, FAS; Nóbrega, LB da; Vieira, DJ; Pereira, JR. 1998b. Efeito de população de plantas no rendimento do consórcio de mamona / sorgo. Revista de Oleaginosas e Fibrosas 2 (3):183-192.

Azevedo, DMP de; Santos, JW dos; Lima, EF; Batista, FAS; Pereira, JR; Vieira, DJ, Nóbrega, LB da, Pereira, JR. 2001. Efeito de população de plantas no consórcio mamoneira/milho. II. Eficiência Agronômica. Revista de Oleaginosas e Fibrosas 5(1):255- 265.

Azevedo, DMP; Santos, JW dos; Santos, TS; Leão, AB. 2006. Período crítico de competição entre mamoneira e plantas daninhas. Revista Brasileira de Oleaginosas e Fibrosas, 10(2): 1017-1024.

Azevedo, DMP de; Beltrão, JWS; Severino, LS; Santos, JW dos; Leão, AB. 2007. Rendimento e eficiência agronômica do consórcio da mamoneira com cereais e feijão caupi no semiárido nordestino. Revista de Oleaginosas e Fibrosas 11(3):145-162.

Beltrão, NEM; Vale, LS; Marques, LF; Cardoso, LS; Maracaja, PB. 2010. Época relativa de plantio no consórcio mamona e gergelim. Revista verde de agroecologia e desenvolvimento sustentável. 5(5):67-73.

Bertoni, J; Lombardi Neto, F; Benatti Jr, R. 1976. Estudos em lisímetros monolíticos, de perdas de agua e evapotranspiração em três tipos de solos sob diferentes condições de uso. Bragantia 35:123-145.

Bezerra Neto, F; Robichaux, RH. 1996. Spatial arrangement and density effects on an annual cotton/cowpea/maize intercrop. I. Agronomic efficiency. Pesquisa Agropecuária Brasileira 31(10): 729-741.

Bezerra Neto, F; Robichaux, RH. 1997. Spatial arrangement and density effects on an annual cotton/cowpea/maize intercrop. II. Yield and biomass. Pesquisa Agropecuária Brasileira 32(10):1029- 1037.

Calegari, A. 2007. Uso adequado de plantas de cobertura, rotação de culturas e seus benefícios no sistema do plantio direto. Londrina, BR; IAPAR. p. 1- 8.

Corrêa, MLP; Tâvora, FJAF; Pitombeira, JB. 2006. Comportamento de cultivares de mamona en sistema de cultivo isolados e consorciados com caupi e sorgo granífero. Revista Ciência Agronómica 37(2):200-207.

Costa, AGF; Sofiatti, V; Maciel, CDG; Poletine, JP; Sousa, JI de. 2014. Weed management strategies for castor bean crops. Acta Scientiarum. Agronomy, 36(2), 137145. Revisado en Mayo 09, 2015, disponible en: http://www.scielo.br/scielo.php?script=sci_arttext\&pid $=$ S1807-86212014000200001\&lng=en\&tlng=en. 10.40 25/actasciagron.v36i2.17090.

DCEA/MAG (Dirección de Censos y Estadísticas Agropecuarias/ Ministerio de Agricultura y Ganadería, PY). 2006. Síntesis estadística. Producción agropecuaria. Año agrícola 2005/2006. San Lorenzo, PY. MAG. 49 p.

DCEA/MAG (Dirección de Censos y Estadísticas Agropecuarias/ Ministerio de Agricultura y Ganadería, PY). 2009. Censo Agropecuario Nacional 2.008. San Lorenzo, PY. MAG. V. II. 493 p.

DCEA/MAG (Dirección de Censos y Estadísticas Agropecuarias/ Ministerio de Agricultura y Ganadería, PY). 2013. Síntesis estadística. Producción agropecuaria 2012/2013. San Lorenzo, PY. MAG. 47 p.

Fatecha A, 2001. Guía para la fertilización de cultivos anuales de la Región Oriental del Paraguay. San Lorenzo, PY. Sociedad Paraguaya de Ciencia del Suelo- SOPACIS, Paraguay. Volumen 1.33 p. 
Flores G, ML \& Maldonado C, LG. 2007. Rentabilidad e incidencia de la producción de tártago (Rícínus comunnís L.) en los ingresos de pequeños productores del distrito de Choré, San Pedro. Revista Investigación Agraria. 9(2):39- 45.

Francelino de O Filho, A; Bezerra FTC; Pitombeira, JB; Barros, GL; Oliveira, F de A. 2013. Contribuição dos racemos na produção de mamoneira em consórcios com culturas alimentícias. Revista Verde. 7(2):114-124.

Furtado, G de F; Sousa A dos S; Sousa Jr, JR, Sousa, FRM; Lacerda SS de A; Silva SS. 2014. Rendimento e correlações da mamoneira consorciada com feijãocaupi e gergelim no semiárido paraibano. Revista Brasileira de Engenharia Agrícola e Ambiental. 18 (9):892-898.

Loncharich, J. 2008. Caracterización Ambiental del Departamento de Paraguarí: Bases para la Planificación del Uso de la Tierra. Tesis (Ing. Agr.). San Lorenzo, PY. Carrera Ingeniería Agronómica. FCA-UNA. 78 p.

Lopez, O; Gonzalez, E; Llamas, PA; Molinas, A; Franco, E; Garcia. S; Rios, E. 1995. Estudio de reconocimiento de suelos, capacidad de uso de la tierra y propuesta de ordenamiento territorial preliminar de la Región Oriental del Paraguay. Asunción, PY: MAG/SRNMA/BM/PRUT. v.1, 197 p.

Magalhães, ID; Soares, CS; Costa, FE; Almeida, AE da S; Oliveira, AB; Vale, LS. 2013. Viabilidade do consórcio mamona-gergelim para a agricultura familiar no semiárido paraibano: Influência de diferentes épocas de plantio Rev. Bras. de Agroecologia. 8(1): 57-65.

Morgado, LB. 2004. Efeito da variação da população de plantas na productividade da mamona em plantio consorciado com sorgo (Shorghum bicolor L.) e caupi (Vigna ungiculata (L.) Walp). In: Congresso Brasileiro de Mamona (1, 2004, Campina Grande, BR). Trabajos presentados. Campina Grande, BR: UFPB/CCA, EMBRAPA/CNPA. 4 p.

Mundomanz. 2014. Datos climáticos. Consultado en 8 de julio del 2014. Disponible en: http://www.mundo manz.com/meteo_p/yearrep?countr=PARAGUAY\&ind $=86221 \&$ year $=2009 \&$ action=display

Paulo, EM; Kasai, FS; Savy Filho, A.1997. Efeitos da largura da faixa de capina na cultura da mamona. Bragantia 51(1):145-153.

Pinto, C de M; Pinto O, OR; Siszenando Filho, FA. 2011. Mamona consorciada com girassol em plantios defasados: eficiência biológica. Revista Verde. 6(5):166-176.

Rando, EM; Quintanilha, AC. 1990. Crescimento e produção da mamona em consórcio com culturas e adubos verdes. Pesquisa Agropecuária Brasileira 25(11):1569-1575.

Rezende, B; Cecílio Filho, AB; Canato, GHD; Martins, MIEG. 2005. Análise econômica de consorciados de alface $\mathrm{x}$ tomate, em cultivo protegido, Jaboticabal-SP. Científica 33(1):42-49.

Savy Filho, A; Carvalho, LO; Banzatto, NV. 1989. Avaliação da produtividade da mamoneira (Ricinus communis L.), cv. IAC-80, em consorciação de culturas. Demonstração de resultados. Revista de Agricultura 64(2):179-193.

Sousa Jr, JR; Furtado, GF; Sousa, JRM; Lacerda, RRA; Souza, A dos S; Maracajá, PB. 2012. Consórcio de mamona com gergelim e feijão-caupi no semiárido paraibano: componentes de produção. 8(3):20-28.

Távora, FJAF; Melo, FIO; Silva, FP da; Barbosa Filho, M. 1988. Consorciação da mamona com culturas anuais de ciclo curto. Ciência Agronômica 19(2):85-94,

Teixeira, IR; Mota, JH; Silva, AG. 2005. Consórcio de hortaliças. Semina: Ciências Agrárias, Londrina, 26(4):507-514.

Teixeira, IR; Silva, GC; Oliveira, JA de P; Timossi, PC. 2012. Arranjos de plantas do feijoeiro-comum consorciado com mamona. Revista Caatinga. 25(2):85-89.

Teixeira, IR; Silva, GC; Timossi, PC; Silva, AG. 2011. Desempenho agronômico de cultivares de feijão-comum consorciado com mamona. Revista Caatinga. 24(4):55-61.

Willey, RW. 1979. Intercropping - its importance and research needs. Part 1. Competition and yield advantagens. Field Crop Abstracts 32(1):1-10.

Zaffaroni, E. 1987. Yield stability of sole and intercropping systems in the northeast of Brazil. Pesquisa Agropecuária Brasileira 22:393-99. 